\title{
On some subclasses of hypergeometric functions with Djrbashian Cauchy type kernel
}

\author{
Joel Esteban Restrepo ${ }^{a}$, Armen Jerbashian ${ }^{a}$, Praveen Agarwal ${ }^{b, *}$ \\ a Institute of Mathematics, University of Antioquia, Cl. 53 - 108, Medellin, Colombia. \\ ${ }^{b}$ Department of Mathematics, Anand International College of Engineering, Jaipur 303012, India. \\ Communicated by Y. J. Cho
}

\begin{abstract}
In this paper, some new integral representations are proved for several weighted hypergeometric functions introduced recently in [J. E. Restrepo, A. K1lıçman, P. Agarwal, O. Altun, Adv. Difference Equ., 2017 (2017), 11 pages]. Besides, some new subclasses of weighted hypergeometric functions containing the Djrbashian Cauchy type kernel are introduced. The series representing the considered hypergeometric functions are convergent out of some sets of zero $\omega$-capacity, and these hypergeometric functions have finite boundary values everywhere on $|z|=1$, out of zero $\omega$-capacity sets. (c)2017 All rights reserved.
\end{abstract}

Keywords: Weighted hypergeometric function, Djrbashian Cauchy type kernel, $\omega$-capacity, boundary behavior. 2010 MSC: 33C20, 31A10, 31A20.

\section{Introduction}

The analysis of extensions and generalizations of hypergeometric functions is a well-known, contemporary investigation field (see, e.g., [1, 12,15-17]). For introducing the considered in this paper weighted hypergeometric functions, we recalled the weighted extension [14] of Euler's beta-function:

$$
B_{\omega}^{(\alpha, \beta)}(x, y)=\int_{0}^{1} t^{x-1}(1-t)^{y-1} \omega^{(\alpha, \beta)}(z t, u, v) d t
$$

where $\operatorname{Re} x>0$, Re $y>0$, while $\alpha, \beta, z, u, v$ are real or complex parameters and $\omega^{(\alpha, \beta)}(z t, u, v)$ is a function of the class $\Omega$, which means that the integral (1.1) is absolutely convergent for $\alpha, \beta, z, u, v$ changing in several, fixed regions. Note that these regions can be different while considering different functions. For instance, if $\omega(t, p, 0)=e^{\frac{-p}{t(1-t)}}$ with Re $p>0$ and $t \in[0,1]$, then $B_{\omega}(x, y)$ becomes the extension of Euler's beta-function considered by Chaudhry et al. [3] (see also the recent paper [14]).

\footnotetext{
*Corresponding author

Email addresses: cocojoe189@yahoo.es (Joel Esteban Restrepo), armen-jerbashian@yahoo.com (Armen Jerbashian), goyal . praveen2011@gmail.com (Praveen Agarwal)

doi:10.22436/jnsa.010.05.06
} 


\section{2. $\omega$-weighted hypergeometric functions}

In this paper, we deal with the weighted versions of the Gauss hypergeometric function ${ }_{2} \mathrm{~F}_{1}$, the Appell hypergeometric function $F_{2}$, the generalized Gauss hypergeometric function $F_{\omega}$ and the generalized confluent hypergeometric function ${ }_{1} \mathrm{~F}_{1}^{\omega}$, which all are introduced in [14]. We shall recall the definitions of these functions, and again assume that $m \in \mathbb{N}, \omega^{(\alpha, \beta)} \in \Omega, B(x, y)$ is the classical beta function with $\operatorname{Re} x, \operatorname{Re} y>0$, and $(\alpha)_{n}$ is the Pochhammer's symbol:

$$
(\alpha)_{n}=\frac{\Gamma(\alpha+n)}{\Gamma(\alpha)}, \quad(\alpha)_{0}=1
$$

Now, let us define our $\omega$-weighted extended hypergeometric functions.

Definition 2.1. The $\omega$-weighted extended Gauss hypergeometric function is

$$
{ }_{2} \mathrm{~F}_{1}(\mathrm{a}, \mathrm{b} ; \mathrm{c} ; z ; \omega):=\sum_{n=0}^{\infty} \frac{(\mathrm{a})_{n}(\mathrm{~b})_{n}}{(\mathrm{~b}-\mathrm{m})_{n}} \frac{\mathrm{B}_{\omega}^{(\alpha, \beta)}(\mathrm{b}-\mathrm{m}+\mathrm{n}, \mathrm{c}-\mathrm{b}+\mathrm{m})}{\mathrm{B}(\mathrm{b}-\mathrm{m}, \mathrm{c}-\mathrm{b}+\mathrm{m})} \frac{z^{\mathrm{n}}}{\mathrm{n} !},
$$

where $|z|<1$ and $m<\operatorname{Re} b<\operatorname{Re} c$.

Definition 2.2. The $\omega$-weighted extended Appell hypergeometric function is

$$
\begin{aligned}
F_{2}(a, b, c ; d, e ; x, y ; \omega): & =\sum_{n, k=0}^{\infty} \frac{(a)_{n+k}(b)_{n}(c)_{k}}{(b-m)_{n}(e)_{k}} \frac{B_{\omega}^{(\alpha, \beta)}(b-m+n, d-b+m)}{B(b-m, d-b+m)} \frac{x^{n}}{n !} \frac{y^{k}}{k !} \\
& =\sum_{n, k=0}^{\infty} \frac{(a)_{n+k}(b)_{n}(c)_{k}}{(d)_{n}(c-m)_{k}} \frac{B_{\omega}^{(\alpha, \beta)}(c-m+k, e-c+m)}{B(c-m, e-c+m)} \frac{x^{n}}{n !} \frac{y^{k}}{k !}
\end{aligned}
$$

where $|x|+|y|<1, m<\operatorname{Re} b<\operatorname{Re} d$, and $m<\operatorname{Re} c<\operatorname{Re} e$.

One can be convinced that the above defined functions become those of [11] for $\omega(t, p, 0)=e^{\frac{-p}{t(1-t)}}$ and Re $p>0$, while for $\omega \equiv 1$ these functions become the well-known Gauss hypergeometric function ${ }_{2} F_{1}$ and the Appell function $F_{2}$, respectively.

Definition 2.3. The $\omega$-weighted generalized Gauss hypergeometric function is

$$
F_{\omega}(a, b ; c ; z)=\sum_{n=0}^{\infty}(a)_{n} \frac{B_{\omega}^{(\alpha, \beta)}(b+n, c-b)}{B(b, c-b)} \frac{z^{n}}{n !}
$$

where $|z|<1$ and $\operatorname{Re} c>\operatorname{Re} b>0$.

Definition 2.4. The $\omega$-weighted generalized confluent hypergeometric function is

$$
{ }_{1} \mathrm{~F}_{1}^{\omega}(b ; c ; z)=\sum_{n=0}^{\infty} \frac{\mathrm{B}_{\omega}^{(\alpha, \beta)}(b+n, c-b)}{B(b, c-b)} \frac{z^{n}}{n !},
$$

where $|z|<1$ and $\operatorname{Re} c>\operatorname{Re} b>0$.

\section{Main results}

In this section, we prove the main results of the present paper. We start by some integral representation theorems.

Theorem 3.1. The following integral representations are true:

$$
\begin{aligned}
& \mathrm{B}(\mathrm{b}-\mathrm{m}, \mathrm{c}-\mathrm{b}+\mathrm{m}){ }_{2} \mathrm{~F}_{1}(\mathrm{a}, \mathrm{b} ; \mathrm{c} ; z ; \omega) \\
& \quad=\int_{0}^{1} \mathrm{t}^{\mathrm{b}-\mathrm{m}-1}(1-\mathrm{t})^{\mathrm{c}-\mathrm{b}+\mathrm{m}-1} \omega^{(\alpha, \beta)}(z \mathrm{t}, \mathrm{u}, v)_{2} \mathrm{~F}_{1}(\mathrm{a}, \mathrm{b} ; \mathrm{b}-\mathrm{m} ; z \mathrm{t}) \mathrm{dt},
\end{aligned}
$$




$$
\begin{gathered}
B(c-m, e-c+m) F_{2}(a, b, c ; d, e ; x, y ; w) \\
=\int_{0}^{1} t^{c-m-1}(1-t)^{e-c+m-1} \omega^{(\alpha, \beta)}(z t, u, v) F_{2}(a, b, c ; d, c-m ; x, y t) d t, \\
F_{\omega}(a, b ; c ; z)=\frac{1}{B(b, c-b)} \int_{0}^{1} t^{b-1}(1-t)^{c-b-1} \omega^{(\alpha, \beta)}(t z, u, v){ }_{2} F_{1}(a, b ; b ; z t) d t
\end{gathered}
$$

where ${ }_{2} \mathrm{~F}_{1}(\mathrm{a}, \mathrm{b} ; \mathrm{c} ; \mathrm{z})$ and $\mathrm{F}_{2}(\mathrm{a}, \mathrm{b}, \mathrm{c} ; \mathrm{d}, \mathrm{e} ; \mathrm{x}, \mathrm{y})$ are defined in $[2, p .1$ and $p p .73-74]$.

Proof. The representations (3.1), (3.2), and (3.3) follow by Definitions 2.1, 2.2, 2.3, and formula (1.1) for the function $B_{\omega}^{(\alpha, \beta)}$.

Before proceeding to the proofs of some other integral representation theorems, we recall some definitions and statements from [6,7].

Everywhere below, a function $\omega(t)$ is said to be of the class $\Omega_{0}$, if

(i) $\omega(t)>0$ and is continuous and nondecreasing in $[0,1)$,

(ii) $\omega(0)=1$ and $\int_{0}^{1} \omega(t) d t<+\infty$,

(iii) $\omega(t)$ satisfies the Lipschitz condition at the point $t=0$.

Djrbashian's Cauchy type kernel (see [7] or [6, p. 76]) is the function

$$
\mathrm{C}_{\omega}(z):=\sum_{\mathrm{k}=0}^{+\infty} \frac{z^{\mathrm{k}}}{\Delta_{\mathrm{k}}}, \quad \Delta_{0}=1, \quad \Delta_{\mathrm{k}}=\mathrm{k} \int_{0}^{1} \mathrm{t}^{\mathrm{k}-1} \omega(\mathrm{t}) \mathrm{dt} \quad(\mathrm{k}=1,2, \ldots) .
$$

Note that for any $\omega(t) \in \Omega_{0}$, the function $C_{\omega}(z)$ is holomorphic in the unit disc $\mathbb{D}=\{z \in \mathbb{C}:|z|<1\}$, and in the particular case $\omega(x)=(1-x)^{\alpha}(-1<\alpha<0)$ it becomes the $1+\alpha$ order of the ordinary Cauchy kernel:

$$
\mathrm{C}_{\omega}(z)=\frac{1}{(1-z)^{1+\alpha}}:=\mathrm{C}_{\alpha}(z),\left.\quad \mathrm{C}_{\omega}(z)\right|_{\omega \equiv 1}=\frac{1}{1-z}, \quad z \in \mathbb{D} .
$$

Besides, the corresponding Schwartz type kernel is

$$
\mathrm{S}_{\omega}(z):=2 \mathrm{C}_{\omega}(z)-1=1+2 \sum_{\mathrm{k}=1}^{+\infty} \frac{z^{\mathrm{k}}}{\Delta_{\mathrm{k}}}, \quad z \in \mathbb{D},
$$

and

$$
\left.S_{\omega}(z)\right|_{\omega(x)=(1-x)^{\alpha}}=\frac{2}{(1-z)^{1+\alpha}}-1:=S_{\alpha}(z),\left.\quad S_{\omega}(z)\right|_{\omega \equiv 1}=\frac{1+z}{1-z}, \quad z \in \mathbb{D} .
$$

Definition 3.2. Let $E \subset[0,2 \pi]$ be a Borel measurable set (B-set) and $\omega \in \Omega_{0}$. It is said that $E$ is of positive $\omega$-capacity, i.e., $C_{\omega}(E)>0$, if there exists a nonnegative $B$-measure $\mu$ supported and finite on $E$ and such that

$$
S_{1} \equiv \lim _{r \rightarrow 1-0} \max _{0 \leqslant \varphi \leqslant 2 \pi} \int_{0}^{2 \pi}\left|C_{\omega}\left(r e^{i(\varphi-\theta)}\right)\right| d \mu(\theta)<+\infty .
$$

If there is no such a measure, i.e., if $S_{1}=\infty$ for any nonnegative B-measure supported on $E$, then $E$ is said to be of zero $\omega$-capacity, i.e., $\mathrm{C}_{\omega}(\mathrm{E})=0$.

One can see that $C_{\omega}(E)=0$, if $E$ is a countable set (see [6, p. 94]). Further, it is easy to see that for $\omega(x)=(1-x)^{\alpha}(-1<\alpha<0)$ the $\omega$-capacity becomes Frostman's well-known $\alpha$-capacity [8-10] (see also $[6,7])$. 
Assuming that $\omega \in \Omega_{0}$, we shall use the following operators (see [5, Lemma 1.1], also [6, 7]) formally defined on functions $u(z)$ given in $\mathbb{D}$ :

$$
\mathrm{L}_{\omega_{1}} u(z):=-\int_{0}^{1} u(z t) d \omega_{1}(t), \quad \text { where } \quad \omega_{1}(t)=\int_{t}^{1} \frac{\omega(x)}{x} d x,
$$

and

$$
\mathrm{L}_{\omega} \mathrm{u}(z)=\mathrm{u}(0)+\mathrm{L}_{\omega_{1}} \mathrm{u}(z), \quad \text { where } \mathrm{U}(z)=|z| \frac{\partial}{\partial|z|} \mathrm{u}(z), \quad z \in \mathbb{D} .
$$

It is not difficult to see that

$$
\begin{aligned}
\mathrm{L}_{\omega}\left[\mathrm{r}^{\mathrm{k}}\right] & =\mathrm{r}^{\mathrm{k}} \Delta_{\mathrm{k}}, \quad \mathrm{r} \in[0,1], \quad \mathrm{k}=0,1,2, \ldots, \\
\left.\mathrm{L}_{\omega} \mathrm{u}(z)\right|_{\mathfrak{u}(z) \equiv 1} & =1, \quad z \in \mathbb{D},
\end{aligned}
$$

besides, by (3.4) and (3.6)

$$
\mathrm{L}_{\omega} \mathrm{C}_{\omega}(z)=\frac{1}{1-z}, \quad z \in \mathbb{D} .
$$

For finding some conditions in the terms of $\omega$-capacity, which provide the convergence of the series representing some of the considered weighted hypergeometric functions, we define two special $\Omega$-sets.

Definition 3.3. Under the assumption that $\left\{z_{n}\right\}_{1}^{\infty} \subset \mathbb{D} \backslash\{0\}$ is an arbitrary sequence numerated in the order of nondecreasing modulus, $\Omega^{*}\left(\left\{z_{n}\right\}_{1}^{\infty}\right)$ is the set of the functions

$$
\omega\left(t, z_{n} e^{-i \theta}, p\right)=\operatorname{Re}\left\{C_{\omega_{1}}\left(z_{n} e^{-i \theta}\right)\right\} e^{\frac{-p}{t(1-t)}}\left(1-\left|z_{n}\right|\right) /(a+1)_{n},
$$

where $n \in \mathbb{N}, \theta \in[0,2 \pi]$, Re $p>0, a \in \mathbb{C} \backslash \mathbb{Z}^{-}\left(\mathbb{Z}^{-}\right.$is the set of negative integers) and $\omega_{1} \in \Omega_{0}$.

For a single point $0 \neq z \in \mathbb{D}, \Omega^{*}(z)$ is the set of the functions

$$
\omega^{(n)}\left(t, z e^{-i \theta}, p\right)=\operatorname{Re}\left\{C_{\omega_{1}}\left(z e^{-i \theta}\right)\right\} e^{\frac{-p}{t(1-t)}} /(a+1)_{n},
$$

where the parameters are as above.

For proving the next theorem, recall the following well-known representation:

$$
B_{p}(x, y)=\int_{0}^{1} t^{x-1}(1-t)^{y-1} e^{\frac{-p}{\mathfrak{t}(1-t)}} d t
$$

where $\operatorname{Re} x>0, \operatorname{Re} y>0$ and $\operatorname{Re} p>0$ (see [11, p. 3611]).

Theorem 3.4. If $\omega \in \Omega^{*}\left(\left\{z_{n}\right\}_{1}^{\infty}\right)$ and $\mathrm{m}<\operatorname{Re} \mathrm{b}<\operatorname{Re} \mathrm{c}$, then for all $\vartheta \in[0,2 \pi]$, except a set $\mathrm{E} \subset[0,2 \pi]$ with $\mathrm{C}_{\omega_{1}}(\mathrm{E})=0$, the sum

$$
{ }_{2} \mathrm{~F}_{1}(\mathrm{a}, \mathrm{b} ; \mathrm{c} ; z ; \omega)=\sum_{n=0}^{\infty} \operatorname{Re}\left\{\mathrm{C}_{\omega_{1}}\left(z_{\mathfrak{n}} e^{-\mathrm{i \theta}}\right)\right\} \frac{\left(1-\left|z_{n}\right|\right)(\mathrm{a})_{\mathfrak{n}}(\mathrm{b})_{\mathfrak{n}}}{(\mathrm{b}-\mathrm{m})_{\mathfrak{n}}(\mathrm{a}+1)_{\mathfrak{n}}} \frac{\mathrm{B}_{\mathrm{p}}(\mathrm{b}-\mathrm{m}+\mathrm{n}, \mathrm{c}-\mathrm{b}+\mathrm{m})}{\mathrm{B}(\mathrm{b}-\mathrm{m}, \mathrm{c}-\mathrm{b}+\mathrm{m})} \frac{z^{\mathrm{n}}}{\mathrm{n} !}
$$

is absolutely and uniformly convergent in the closed unit disc $|z| \leqslant 1$.

Proof. Note that by Definition 2.1 in [11] for any $m<\operatorname{Re} b<\operatorname{Re} c$, the series

$$
{ }_{2} F_{1}(a, b ; c ; z ; p)=\sum_{n=0}^{\infty} \frac{(a)_{n}(b)_{n}}{(b-m)_{n}} \frac{B_{p}(b-m+n, c-b+m)}{B(b-m, c-b+m)} \frac{z^{n}}{n !}
$$

is absolutely and uniformly convergent in $|z|<1$. Hence, the sum

$$
\sum_{n=0}^{\infty} \frac{\left(1-\left|z_{n}\right|\right)(a)_{n}(b)_{n}}{(b-m)_{n}(a+1)_{n}} \frac{B_{p}(b-m+n, c-b+m)}{B(b-m, c-b+m)} \frac{z^{n}}{n !}
$$

is absolutely and uniformly convergent in $|z|<1$. Further, one can see that the modulus of $B_{p}(b-m+$ 
$n, c-b+m)$ is bounded by the classical beta-function $B(b-m+n, c-b+m)$. Besides,

$$
\Gamma(z)=\lim _{n \rightarrow+\infty} \frac{n ! n^{z-1}}{(z)_{n}} .
$$

Hence, the following asymptotic relation is true:

$$
\begin{aligned}
\frac{(a)_{n}(b)_{n}}{(b-m)_{n}(a+1)_{n} n !} B(b-m+n, c-b+m) & =\frac{\Gamma(b-m) \Gamma(c-b+m)}{\Gamma(c)} \frac{(a)_{n}(b)_{n}}{(c)_{n}(a+1)_{n} n !} \\
& \approx \frac{\Gamma(b-m) \Gamma(c-b+m)}{\Gamma ! n^{a-1}} \frac{n ! n^{b-1}}{\Gamma(a)} \frac{\Gamma(c)}{\Gamma(b)} \frac{\Gamma(a+1)}{n ! n^{c-1}} \frac{1}{n ! n^{a}} \frac{1}{n !} \\
& =\frac{\Gamma(b-m) \Gamma(c-b+m) a}{\Gamma(b)} \frac{1}{n^{c-b+1} n !} \text { as } n \rightarrow \infty .
\end{aligned}
$$

Consequently, for $|z|=1$ and some constant $0<M<+\infty$,

$$
\begin{aligned}
\left|\sum_{n=1}^{\infty} \frac{\left(1-\left|z_{n}\right|\right)(a)_{n}(b)_{n}}{(b-m)_{n}(a+1)_{n}} \frac{B_{p}(b-m+n, c-b+m)}{B(b-m, c-b+m)} \frac{z^{n}}{n !}\right| \\
\leqslant M\left|\frac{\Gamma(b-m) \Gamma(c-b+m) a}{\Gamma(b)}\right| \sum_{n=1}^{\infty} \frac{1}{n^{1+\operatorname{Re}\{c-b\}}<+\infty,}
\end{aligned}
$$

since $\operatorname{Re} c-\operatorname{Re} b>0$. Now, the desired result holds by Theorem 2.2 in [6, p. 94].

Theorem 3.5. If $\omega \in \Omega^{*}\left(\left\{z_{n}\right\}_{1}^{\infty}\right)$ and $0<\operatorname{Re} b<\operatorname{Re} c$, then for all $\vartheta \in[0,2 \pi]$, except a set $\mathrm{E} \subset[0,2 \pi]$ with $\mathrm{C}_{\omega_{1}}(\mathrm{E})=0$, the sum

$$
\mathrm{F}_{\omega}(\mathrm{a}, \mathrm{b} ; \mathrm{c} ; z)=\sum_{n=0}^{\infty} \operatorname{Re}\left\{\mathrm{C}_{\omega_{1}}\left(z_{n} e^{-i \theta}\right)\right\} \frac{\left(1-\left|z_{n}\right|\right)(\mathrm{a})_{n}}{(\mathrm{a}+1)_{n}} \frac{\mathrm{B}_{\mathrm{p}}(\mathrm{b}+\mathrm{n}, \mathrm{c}-\mathrm{b})}{\mathrm{B}(\mathrm{b}, \mathrm{c}-\mathrm{b})} \frac{z^{\mathrm{n}}}{\mathrm{n} !}
$$

is absolutely and uniformly convergent in $|z| \leqslant 1$.

Proof. In [4] (see also [13]) it is proved that for $\operatorname{Re} p>0$ and $\operatorname{Re} c>\operatorname{Re} b>0$, the series

$$
\mathrm{F}_{\mathrm{p}}(\mathrm{a}, \mathrm{b} ; \mathrm{c} ; z)=\sum_{n=0}^{\infty}(\mathrm{a})_{n} \frac{\mathrm{B}_{\mathrm{p}}(\mathrm{b}+\mathrm{n}, \mathrm{c}-\mathrm{b})}{\mathrm{B}(\mathrm{b}, \mathrm{c}-\mathrm{b})} \frac{z^{\mathrm{n}}}{\mathrm{n} !}
$$

is absolutely and uniformly convergent in $|z|<1$. Hence, also the sum

$$
\sum_{n=0}^{\infty} \frac{\left(1-\left|z_{n}\right|\right)(a)_{n}}{(a+1)_{n}} \frac{B_{p}(b+n, c-b)}{B(b, c-b)} \frac{z^{n}}{n !}
$$

is absolutely and uniformly convergent in $|z|<1$. Besides, the modulus of $B_{p}(b+n, c-b)$ is bounded by $B(b+n, c-b)$, and hence

$$
\begin{aligned}
\frac{(a)_{n}}{(a+1)_{n}} B(b+n, c-b) \frac{1}{n !} & =\frac{\Gamma(c-b) \Gamma(b)}{\Gamma(c)} \frac{(a)_{n}(b)_{n}}{(a+1)_{n}(c)_{n}} \frac{1}{n !} \\
& \approx \frac{\Gamma(c-b) \Gamma(b)}{\Gamma(c)} \frac{n ! n^{a-1}}{\Gamma(a)} \frac{n ! n^{b}-1}{\Gamma(b)} \frac{\Gamma(a+1)}{n ! n^{a}} \frac{\Gamma(c)}{n ! n^{c-1}} \frac{1}{n !}=\frac{\Gamma(c-b) a}{\Gamma(a)} \frac{1}{n^{c-b+1} n !} .
\end{aligned}
$$

Consequently, the series

$$
\sum_{n=0}^{\infty} \frac{\left(1-\left|z_{n}\right|\right)(a)_{n}}{(a+1)_{n}} \frac{B_{p}(b+n, c-b)}{B(b, c-b)} \frac{z^{n}}{n !}
$$

is absolutely and uniformly convergent in $|z| \leqslant 1$, and the desired result follows by Theorem 2.2 in [6]. 
Remark 3.6. In the same way, the statement of Theorem 3.5 can be proved for the $\omega$-weighted generalized confluent hypergeometric function ${ }_{1} \mathrm{~F}_{1}^{\omega}$ with $\operatorname{Re} \mathrm{a}>1$.

Remark 3.7. In formula (3.7), the factor $(a+1)_{\mathfrak{n}}$ in the denominator helps to prove the statement on the convergence over $|z|=1$. Obviously the convergences of Theorems 3.4 and 3.5 in $|z|<1$ can be proved in absence of $(a+1)_{n}$ in denominators of the sums.

The next two theorems establish that the functions ${ }_{2} F_{1}(a, b ; c ; z ; \omega), F_{\omega}(a, b ; c ; z)$, and ${ }_{1} F_{1}^{\omega}$ belong to the class $U_{\omega}\left(\omega \in \Omega_{0}\right)$ defined in [6, p. 37], which permits to write them as some Schwarz type integrals with measures of bounded variation.

Theorem 3.8. If $\omega \in \Omega^{*}\left(\left\{z_{n}\right\}_{1}^{\infty}\right)$ and $m<\operatorname{Re} b<\operatorname{Re} c$, then

$$
{ }_{2} \mathrm{~F}_{1}\left(\mathrm{a}, \mathrm{b} ; \mathrm{c} ; \mathrm{re} \mathrm{i}^{i \varphi} ; \omega\right)=\frac{1}{2 \pi} \int_{0}^{2 \pi} \operatorname{Re} S_{\omega}\left(r \mathrm{r}^{i(\varphi-\lambda)}\right) \mathrm{d} \sigma(\lambda), \quad r \in[0,1), \varphi \in[0,2 \pi],
$$

where $\sigma(\lambda)$ is a function of bounded variation on $[0,2 \pi]$ and some finite non-tangential boundary values

$$
\lim _{z \rightarrow e^{i \varphi}}{ }_{2} F_{1}(a, b ; c ; z ; w)
$$

exist for all $\varphi \in[0,2 \pi]$, except a set of zero $\omega$-capacity.

Proof. Applying the operator $\mathrm{L}_{\omega}\left(\omega \in \Omega_{0}\right)$ to the representation (3.7) of ${ }_{2} \mathrm{~F}_{1}(\mathrm{a}, \mathrm{b} ; \mathrm{c} ; z ; \omega)$ and using formula (3.6), we obtain

$$
\mathrm{L}_{\omega}\left[{ }_{2} \mathrm{~F}_{1}(\mathrm{a}, \mathrm{b} ; \mathrm{c} ; z ; \omega)\right]=\sum_{n=0}^{\infty} \operatorname{Re}\left\{\mathrm{C}_{\omega_{1}}\left(z_{n} e^{-\mathrm{i} \theta}\right)\right\} \frac{\left(1-\left|z_{n}\right|\right)(\mathrm{a})_{n}(\mathrm{~b})_{\mathfrak{n}}}{(\mathrm{b}-\mathrm{m})_{n}(\mathrm{a}+1)_{n}} \frac{\mathrm{B}_{\mathrm{p}}(\mathrm{b}-\mathrm{m}+\mathrm{n}, \mathrm{c}-\mathrm{b}+\mathrm{m})}{\mathrm{B}(\mathrm{b}-\mathrm{m}, \mathrm{c}-\mathrm{b}+\mathrm{m})} \frac{z^{\mathrm{n}} \Delta_{n}}{\mathrm{n} !} .
$$

Hence, similar to the proof of Theorem 3.4 we get

$$
\begin{aligned}
& \left|\int_{0}^{2 \pi} \mathrm{L}_{\omega}\left[{ }_{2} \mathrm{~F}_{1}\left(\mathrm{a}, \mathrm{b} ; \mathrm{c} ; \mathrm{re}^{i \varphi} ; \omega\right)\right] \mathrm{d} \varphi\right| \\
& \quad \leqslant 2 \pi \int_{0}^{1} \omega(\mathrm{t}) \mathrm{dt} \sum_{n=0}^{\infty}\left|\frac{(\mathrm{a})_{n}(\mathrm{~b})_{n}}{(\mathrm{~b}-\mathrm{m})_{n}(\mathrm{a}+1)_{n}} \frac{\mathrm{B}_{p}(\mathrm{~b}-\mathrm{m}+\mathrm{n}, \mathrm{c}-\mathrm{b}+\mathrm{m})}{\mathrm{B}(\mathrm{b}-\mathrm{m}, \mathrm{c}-\mathrm{b}+\mathrm{m})}\right| \frac{1}{(\mathrm{n}-1) !}<+\infty .
\end{aligned}
$$

Thus, ${ }_{2} F_{1}(a, b ; c ; r ; \omega)$ belongs to the class $U_{\omega}$, which by Theorem 1.5 in $[6, p .37]$ implies the representation (3.9). Besides, (3.5) and Theorem 2.5 in [6, p. 112] imply that ${ }_{2} F_{1}(a, b ; c ; z ; \omega)$ has finite non-tangential boundary values for all $\vartheta \in[0,2 \pi]$, except a set of zero $\omega$-capacity.

Theorem 3.9. If $\omega \in \Omega^{*}\left(\left\{z_{n}\right\}_{1}^{\infty}\right)$ and $0<\operatorname{Re} b<\operatorname{Re} c$, then

$$
F_{\omega}\left(a, b ; c ; r e^{\varphi}\right)=\frac{1}{2 \pi} \int_{0}^{2 \pi} \operatorname{Re} S_{\omega}\left(r e^{i(\varphi-\lambda)}\right) d \sigma(\lambda), \quad r \in[0,1), \varphi \in[0,2 \pi],
$$

where $\sigma(\lambda)$ is a function of bounded variation on $[0,2 \pi]$, and some finite non-tangential boundary values

$$
\lim _{z \rightarrow e^{i \varphi}} F_{\omega}\left(a, b ; c ; r e^{\varphi}\right)
$$

exist for all $\varphi \in[0,2 \pi]$, except a set of zero $\omega$-capacity.

Proof. Applying the operator $\mathrm{L}_{\omega}$ with $\omega \in \Omega_{0}$ to the representation (3.8) and formula (3.6), we get

$$
\mathrm{L}_{\omega}\left[\mathrm{F}_{\omega}(\mathrm{a}, \mathrm{b} ; \mathrm{c} ; z)\right]=\sum_{n=0}^{\infty} \operatorname{Re}\left\{\mathrm{C}_{\omega_{1}}\left(z_{n} e^{-i \theta}\right)\right\} \frac{\left(1-\left|z_{n}\right|\right)(\mathrm{a})_{n}}{(\mathrm{a}+1)_{n}} \frac{\mathrm{B}_{\mathrm{p}}(\mathrm{b}+\mathrm{n}, \mathrm{c}-\mathrm{b})}{\mathrm{B}(\mathrm{b}, \mathrm{c}-\mathrm{b})} \frac{z^{\mathrm{n}} \Delta_{\mathrm{n}}}{\mathrm{n} !} .
$$


Consequently, as in the proof of Theorem 3.5 we get

$$
\left|\int_{0}^{2 \pi} L_{\omega}\left[F_{\omega}\left(a, b ; c ; r e^{i \varphi}\right)\right] d \varphi\right| \leqslant 2 \pi \int_{0}^{1} \omega(t) d t \sum_{n=0}^{\infty}\left|\frac{(a)_{n}}{(a+1)_{n}} \frac{B_{p}(b+n, c-b)}{B(b, c-b)}\right| \frac{1}{(n-1) !}<+\infty .
$$

Therefore, $F_{\omega}(a, b ; c ; z)$ belongs to the class $U_{\omega}$, and hence the representation (3.10) follows by Theorem 1.5 in [6]. Further, the existence of the finite non-tangential boundary values for all $\varphi \in[0,2 \pi]$, except a set of zero $\omega$-capacity follows by Theorem 2.5 in [6].

Remark 3.10. Using Remark 3.6, an analog of Theorem 3.9 can be established for the function ${ }_{1} \mathrm{~F}_{1}^{\omega}$.

The next two theorems give a boundary property of some integrals of the functions ${ }_{2} F_{1}(a, b ; c ; z ; \omega)$ and $F_{\omega}(a, b ; c ; z)$, which are in the terms of $\omega$-capacity.

Theorem 3.11. If $\omega \in \Omega^{*}(z), \mathrm{m}<\operatorname{Re} \mathrm{b}<\operatorname{Re} \mathrm{c}$ and $\sigma(\theta)$ is an absolutely continuous measure on [0,2 $\left.\pi\right]$, then the integral

$$
{ }_{2} \mathrm{IF}_{1}\left(\mathrm{a}, \mathrm{b} ; \mathrm{c} ; r \mathrm{e}^{\varphi} ; \omega\right):=\frac{1}{2 \pi} \int_{0}^{2 \pi}{ }_{2} \mathrm{~F}_{1}\left(\mathrm{a}, \mathrm{b} ; \mathrm{c} ; r \mathrm{e}^{i \varphi} ; \omega^{(n)}\left(\mathrm{t}, \mathrm{re} e^{i(\varphi-\vartheta)}, \mathrm{p}\right)\right) \mathrm{d} \sigma(\theta), \quad 0<\mathrm{r} \leqslant 1,
$$

has finite boundary values for all $\varphi \in[0,2 \pi]$, except a set $\mathrm{E} \subset[0,2 \pi]$ of zero $\omega_{1}$-capacity.

Proof. Note that for $z=r e^{i \varphi}(0<r \leqslant 1, \varphi \in[0,2 \pi])$,

$$
{ }_{2} \mathrm{IF}_{1}(\mathrm{a}, \mathrm{b} ; \mathrm{c} ; z ; \omega)=\frac{1}{2 \pi} \int_{0}^{2 \pi} \operatorname{Re}\left\{\mathrm{C}_{\omega_{1}}\left(z e^{-i \theta}\right)\right\} \mathrm{d} \sigma(\theta) \times \sum_{n=0}^{\infty} \frac{(\mathrm{a})_{\mathfrak{n}}(\mathrm{b})_{\mathfrak{n}}}{(\mathrm{b}-\mathrm{m})_{\mathrm{n}}(\mathrm{a}+1)_{\mathrm{n}}} \frac{\mathrm{B}_{\mathrm{p}}(\mathrm{b}-\mathrm{m}+\mathrm{n}, \mathrm{c}-\mathrm{b}+\mathrm{m})}{\mathrm{B}(\mathrm{b}-\mathrm{m}, \mathrm{c}-\mathrm{b}+\mathrm{m})} \frac{z^{\mathrm{n}}}{\mathrm{n} !} .
$$

By Theorem 3.4, this series is absolutely and uniformly convergent in $|z| \leqslant 1$ (since $|1-| z_{n}|| \leqslant 2, n \in \mathbb{N}$ ) and the integral is defined for all $\vartheta \in[0,2 \pi]$, except a set $E_{1} \subset[0,2 \pi]$ with $C_{\omega_{1}}\left(E_{1}\right)=0$. As $\sigma(\theta)$ is absolutely continuous on $[0,2 \pi]$, by Theorem 2.5 in [6] we get

$$
\lim _{\mathrm{r} \rightarrow 1-0} \frac{1}{2 \pi} \int_{0}^{2 \pi} \operatorname{Re}\left\{\mathrm{C}_{\omega_{1}}\left(r e^{i(\varphi-\theta)}\right)\right\} d \sigma(\theta)=\frac{1}{2 \pi} \int_{0}^{2 \pi} \operatorname{Re}\left\{\mathrm{C}_{\omega_{1}}\left(e^{i(\varphi-\theta)}\right)\right\} d \sigma(\theta),
$$

for any $\varphi \in[0,2 \pi]$, except a set $E_{2} \subset[0,2 \pi]$ of zero $\omega_{1}$-capacity. This completes the proof, since the equalities $C_{\omega_{1}}\left(E_{1}\right)=0$ and $C_{\omega_{1}}\left(E_{2}\right)=0$ imply $C_{\omega_{1}}\left(E_{1} \cup E_{2}\right)=0$.

Theorem 3.12. If $\omega \in \Omega^{*}(z), 0<\operatorname{Re} b<\operatorname{Re} c$ and $\sigma(\theta)$ is an absolutely continuous measure on $[0,2 \pi]$, then the integral

$$
\operatorname{IF}_{\omega}\left(a, b ; c ; r e^{\varphi}\right):=\frac{1}{2 \pi} \int_{0}^{2 \pi} F_{\omega(\theta)}\left(a, b ; c ; r e^{i \varphi}\right) d \sigma(\theta), 0<r \leqslant 1,
$$

has finite boundary values for all $\varphi \in[0,2 \pi]$, except a set $\mathrm{E} \subset[0,2 \pi]$ of zero $\omega_{1}$-capacity.

Proof. It is easy to verify that

$$
\operatorname{IF}_{\omega}(a, b ; c ; z)=\frac{1}{2 \pi} \int_{0}^{2 \pi} \operatorname{Re}\left\{C_{\omega_{1}}\left(z e^{-i \theta}\right)\right\} d \sigma(\theta) \times \sum_{n=0}^{\infty} \frac{(a)_{n}}{(a+1)_{n}} \frac{B_{p}(b+n, c-b)}{B(b, c-b)} \frac{z^{n}}{n !}, z=r e^{i \varphi} .
$$

By Theorem 3.5 this series is absolutely and uniformly convergent on $|z| \leqslant 1$ (since $|1-| z_{n}|| \leqslant 2, n \in \mathbb{N}$ ) and the integral is well-defined for all $\vartheta \in[0,2 \pi]$, except a set $E_{1} \subset[0,2 \pi]$ with $C_{\omega_{1}}\left(E_{1}\right)=0$. Consequently, the proof holds by the absolute continuity of $\sigma(\theta)$, since by Theorem 2.5 in [6] the boundary values of the above integral exist, except a set with $C_{\omega_{1}}\left(E_{2}\right)=0$, and hence $C_{\omega_{1}}\left(E_{1} \cup E_{2}\right)=0$.

Remark 3.13. In the same way as Theorem 3.12 is proved, using Remark 3.6 one can prove that $\mathrm{I}_{1} \mathrm{~F}_{1}^{\omega}$ has finite boundary values for all $\varphi \in[0,2 \pi]$, except a set $E \subset[0,2 \pi]$ of zero $\omega_{1}$-capacity. 


\section{Further results, observations and special cases}

In this section we prove a convergence theorem similar to Theorems 3.4 and 3.5. Then, in Corollaries 4.2-4.5 we observe some special cases of the main results of the paper.

The following theorem proves that the extended Gauss hypergeometric function introduced in [11] is absolutely and uniformly convergent in the unit disc, and even on its boundary, except a set of zero $\omega$-capacity.

Theorem 4.1. If $\omega \in \Omega^{*}\left(\left\{z_{n}\right\}_{1}^{\infty}\right)$ and there is some number $0<\rho<1$ such that $\operatorname{Re}\left\{C_{\omega_{1}}\left(z_{n} e^{-i \theta}\right)\right\} \geqslant \rho>0$ for any $\mathrm{n} \in \mathbb{N}$ and $\theta \in[0,2 \pi]$, and $\mathrm{m}<\operatorname{Re} \mathrm{b}<\operatorname{Re} \mathrm{c}$, then the sum

$$
{ }_{2} \mathrm{~F}_{1}(\mathrm{a}, \mathrm{b} ; \mathrm{c} ; z ; \mathrm{p})=\sum_{n=0}^{\infty} \frac{(\mathrm{a})_{\mathrm{n}}(\mathrm{b})_{n}}{(\mathrm{~b}-\mathrm{m})_{n}} \frac{\mathrm{B}_{\mathrm{p}}(\mathrm{b}-\mathrm{m}+\mathrm{n}, \mathrm{c}-\mathrm{b}+\mathrm{m})}{\mathrm{B}(\mathrm{b}-\mathrm{m}, \mathrm{c}-\mathrm{b}+\mathrm{m})} \frac{z^{\mathrm{n}}}{\mathrm{n} !}, \quad z=r e^{i \theta},
$$

is absolutely and uniformly convergent on $|z| \leqslant 1$ for all $\vartheta \in[0,2 \pi]$, except a set $\mathrm{E} \subset[0,2 \pi]$ with $\mathrm{C}_{\omega_{1}}(\mathrm{E})=0$.

Proof. Note that for any $0 \leqslant r<1$ and $\vartheta \in[0,2 \pi]$

$$
\begin{aligned}
\left|{ }_{2} F_{1}\left(a, b ; c ; r e^{i \theta} ; p\right)\right| & \leqslant \sum_{n=0}^{\infty}\left|\frac{(a)_{n}(b)_{n}}{(b-m)_{n}} \frac{B_{p}(b-m+n, c-b+m)}{B(b-m, c-b+m)} \frac{\left(r e^{i \theta}\right)^{n}}{n !}\right| \\
& \leqslant \frac{1}{\rho} \sum_{n=0}^{\infty} \operatorname{Re}\left\{C_{\omega_{1}}\left(z_{n} e^{-i \theta}\right)\right\}\left|\frac{(a)_{n}(b)_{n}}{(b-m)_{n}} \frac{B_{p}(b-m+n, c-b+m)}{B(b-m, c-b+m)}\right| \frac{r^{n}}{n !} .
\end{aligned}
$$

Since $|1-| z_{n}|| \leqslant 2$ and $n \in \mathbb{N}$, by Theorem 3.4 and Remark 3.7, the last series is absolutely and uniformly convergent for any $0 \leqslant r \leqslant 1$ and $\vartheta \in[0,2 \pi]$, except a set $E \subset[0,2 \pi]$ with $C_{\omega_{1}}(E)=0$.

The next four corollaries hold by setting $\omega_{1}(x)=1$ and $\omega_{1}(x)=(1-x)^{\alpha}(-1<\alpha<0)\left(\omega_{1} \in \Omega_{0}\right.$ in both cases) in Definition 3.3 and Theorems 3.4, 3.5, 3.8, 3.9.

Corollary 4.2. If $\omega \in \Omega^{*}\left(\left\{z_{n}\right\}_{1}^{\infty}\right), m<\operatorname{Re} b<\operatorname{Re} c$, and $z_{n}=r_{n} e^{i \varphi_{n}}$, then the series

$$
{ }_{2} \mathrm{~F}_{1}(\mathrm{a}, \mathrm{b} ; \mathrm{c} ; z ; \omega)=\sum_{n=0}^{\infty} \frac{1-\mathrm{r}_{n} \cos \left(\theta-\varphi_{n}\right)}{\left|1-z_{n} e^{-i \theta}\right|^{2}} \frac{\left(1-\left|z_{n}\right|\right)(a)_{n}(b)_{n}}{(b-m)_{n}(a+1)_{n}} \frac{B_{p}(b-m+n, c-b+m)}{B(b-m, c-b+m)} \frac{z^{n}}{n !}
$$

and

$$
{ }_{2} \mathrm{~F}_{1}(\mathrm{a}, \mathrm{b} ; \mathrm{c} ; z ; \omega)=\sum_{n=0}^{\infty} \frac{\operatorname{Re}\left\{\left(1-\mathrm{r}_{n} e^{i\left(\theta-\varphi_{n}\right)}\right)^{(1+\alpha)}\right\}}{\mid 1-z_{n} e^{-\left.i \theta\right|^{2(1+\alpha)}}} \frac{\left(1-\left|z_{n}\right|\right)(a)_{n}(b)_{n}}{(b-m)_{n}(a+1)_{n}} \frac{B_{p}(b-m+n, c-b+m)}{B(b-m, c-b+m)} \frac{z^{n}}{n !}
$$

are absolutely and uniformly convergent in $|z| \leqslant 1$ for all $\vartheta \in[0,2 \pi]$, except some sets $E_{1}, E_{2} \subset[0,2 \pi]$ with $\mathrm{C}_{\alpha}\left(\mathrm{E}_{1}\right)=\mathrm{C}_{\alpha}\left(\mathrm{E}_{2}\right)=0$, where $\mathrm{C}_{\alpha}$ is Frostman's $\alpha$-capacity.

Corollary 4.3. If $\omega \in \Omega^{*}\left(\left\{z_{n}\right\}_{1}^{\infty}\right), 0<\operatorname{Re} b<\operatorname{Re} c$, and $z_{n}=r_{n} e^{i \varphi_{n}}$, then the series

$$
\mathrm{F}_{\omega}(\mathrm{a}, \mathrm{b} ; \mathrm{c} ; z)=\sum_{n=0}^{\infty} \frac{1-\mathrm{r}_{\mathrm{n}} \cos \left(\theta-\varphi_{n}\right)}{\left|1-z_{n} e^{-i \theta}\right|^{2}} \frac{\left(1-\left|z_{n}\right|\right)(a)_{n}}{(a+1)_{n}} \frac{B_{p}(b+n, c-b)}{B(b, c-b)} \frac{z^{n}}{n !}
$$

and

$$
\mathrm{F}_{\omega}(\mathrm{a}, \mathrm{b} ; \mathrm{c} ; z)=\sum_{n=0}^{\infty} \frac{\operatorname{Re}\left\{\left(1-\mathrm{r}_{\mathrm{n}} \mathrm{e}^{\mathrm{i}\left(\theta-\varphi_{\mathrm{n}}\right)}\right)^{(1+\alpha)}\right\}}{\left|1-z_{n} e^{-i \theta}\right|^{2(1+\alpha)}} \frac{\left(1-\left|z_{n}\right|\right)(\mathrm{a})_{n}}{(\mathrm{a}+1)_{n}} \frac{\mathrm{B}_{\mathrm{p}}(\mathrm{b}+\mathrm{n}, \mathrm{c}-\mathrm{b})}{\mathrm{B}(\mathrm{b}, \mathrm{c}-\mathrm{b})} \frac{z^{\mathrm{n}}}{\mathrm{n} !}
$$

are absolutely and uniformly convergent in $|z| \leqslant 1$ for all $\vartheta \in[0,2 \pi]$, except some sets $E_{1}, E_{2} \subset[0,2 \pi]$ with $\mathrm{C}\left(\mathrm{E}_{1}\right)=\mathrm{C}_{\alpha}\left(\mathrm{E}_{2}\right)=0$. 
The last two corollaries give some representations of the functions ${ }_{2} F_{1}$ and $F_{\omega}$ by the Poisson integral.

Corollary 4.4. If $\omega \in \Omega^{*}\left(\left\{z_{n}\right\}_{1}^{\infty}\right)$ and $\mathrm{m}<\operatorname{Re} \mathrm{b}<\operatorname{Re} \mathrm{c}$, then

$$
{ }_{2} \mathrm{~F}_{1}\left(\mathrm{a}, \mathrm{b} ; \mathrm{c} ; \mathrm{re} \mathrm{e}^{\mathrm{i} \varphi} ; \omega\right)=\frac{1}{2 \pi} \int_{0}^{2 \pi} \frac{1-\mathrm{r}^{2}}{\mid 1-r e^{\left.i(\varphi-\lambda)\right|^{2}}} \mathrm{~d} \sigma(\lambda), \quad r \in[0,1), \varphi \in[0,2 \pi],
$$

where $\sigma(\lambda)$ is a function of bounded variation on $[0,2 \pi]$ and some finite non-tangential boundary values

$$
\lim _{z \rightarrow e^{i \varphi}}{ }_{2} F_{1}\left(a, b ; c ; r e^{i \varphi} ; w\right)
$$

exist for all $\varphi \in[0,2 \pi]$, except a set of zero $\alpha$-capacity.

Corollary 4.5. If $\omega \in \Omega^{*}\left(\left\{z_{n}\right\}_{1}^{\infty}\right)$ and $0<\operatorname{Re} b<\operatorname{Re} c$, then

$$
\mathrm{F}_{\omega}\left(\mathrm{a}, \mathrm{b} ; \mathrm{c} ; r \mathrm{e}^{\varphi}\right)=\frac{1}{2 \pi} \int_{0}^{2 \pi} \operatorname{Re} \frac{1-\mathrm{r}^{2}}{\left|1-r e^{i(\varphi-\lambda)}\right|^{2}} \mathrm{~d} \sigma(\lambda), \quad r \in[0,1), \varphi \in[0,2 \pi],
$$

where $\sigma(\lambda)$ is a function of bounded variation on $[0,2 \pi]$ and some finite non-tangential boundary values

$$
\lim _{z \rightarrow e^{i \varphi}} F_{\omega}\left(a, b ; c ; r e^{\varphi}\right)
$$

exist for all $\varphi \in[0,2 \pi]$, except a set of zero $\alpha$-capacity.

\section{Conclusions}

Some new classes of weighted hypergeometric functions containing Djrbashian's Cauchy type kernel can be used as a bridge to connect a branch of the theory of special functions to the apparatus of the factorization theory of Dzhrbashyan and Zakharyan [6,7]. The delicate notion of $\omega$-capacity, which becomes Frostman's $\alpha$-capacity in a particular case, can be used to describe the sets of rays, where the series representing various general classes of weighted hypergeometric functions are convergent. Some class of such hypergeometric functions can be written as Poisson integrals after application of the operator $\mathrm{L}_{\omega}$, which implies a description of the boundary values on $|z|=1$ by $\omega$-capacities. Besides, it is possible to describe the boundary behavior of the integrals of some other classes of general, weighted hypergeometric functions in the terms of $\omega$-capacity by means of some other techniques. The general results of the paper possess a variety of interesting special cases.

\section{Acknowledgment}

The authors would like to express profound gratitude to referees for deeper review of this paper and the referee's useful suggestions that led to an improved presentation of the paper.

\section{References}

[1] P. Agarwal, J.-S. Choi, K. B. Kachhia, J. C. Prajapati, H. Zhou, Some integral transforms and fractional integral formulas for the extended hypergeometric functions, Commun. Korean Math. Soc., 31 (2016), 591-601. 1

[2] W. N. Bailey, Generalized hypergeometric series, Cambridge Tracts in Mathematics and Mathematical Physics, Stechert-Hafner, Inc., New York, (1964). 3.1

[3] M. A. Chaudhry, A. Qadir, M. Rafique, S. M. Zubair, Extension of Euler's beta function, J. Comput. Appl. Math., 78 (1997), 19-32. 1

[4] M. A. Chaudhry, A. Qadir, H. M. Srivastava, R. B. Paris, Extended hypergeometric and confluent hypergeometric functions, Appl. Math. Comput., 159 (2004), 589-602. 3

[5] A. M. Dzhrbashyan, An extension of the factorization theory of M. M. Dzhrbashyan, (Russian); translated from Izv. Nats. Akad. Nauk Armenii Mat., 30 (1995), 47-75, J. Contemp. Math. Anal., 30 (1995), 39-61. 3 
[6] M. M. Dzhrbashyan, V. S. Zakharyan, Klassy i granichnye svoŭstva funktsiŭ, meromorfnykh v kruge, (Russian) [[Classes and boundary properties of functions that are meromorphic in the disk]] Fizmatlit "Nauka", Moscow, (1993). 3, $3,3,3,3,3,3,3,5$

[7] M. M. Džrbašjan, Theory of factorization and boundary properties of functions meromorphic in the disk, Proceedings of the International Congress of Mathematician, Vancouver, B. C., (1974), Canad. Math. Congress, Montreal, Que., 2 (1975), 197-202. 3, 3, 5

[8] O. Frostman, Potentiel d'équilibre et capacité des ensembles avec quelques applications a la théorie des fonctions, (French) Madd. Lunds. Univ. Mat. Sem., 3 (1935), 1-11. 3

[9] O. Frostman, Sur les produits de Blaschke, Fysiogr. Säldsk. Lund, föhr., 12 (1939), 1-14.

[10] O. Frostman, Sur les produits de Blaschke, (French) Kungl. Fysiografiska Sällskapets i Lund Förhandlingar [Proc. Roy. Physiog. Soc. Lund], 12 (1942), 169-182. 3

[11] İ. O. Kiymaz, A. Çetinkaya, P. Agarwal, An extension of Caputo fractional derivative operator and its applications, J. Nonlinear Sci. Appl., 9 (2016), 3611-3621. 2, 3, 3, 4

[12] L. K. B. Kuroda, A. V. Gomes, R. Tavoni, P. F. de Arruda Mancera, N. Varalta, R. de Figueiredo Camargo, Unexpected behavior of Caputo fractional derivative, Comput. Appl. Math., (2016), 1-11. 1

[13] E. Özergin, Some properties of hypergeometric functions, Ph.D. Thesis, Eastern Mediterranean University, North Cyprus, Turkey, (2011). 3

[14] J. E. Restrepo, A. Kılıçman, P. Agarwal, O. Altun, Weighted hypergeometric functions and fractional derivative, Adv. Difference Equ., 2017 (2017), 11 pages. 1, 1, 2

[15] V. E. Tarasov, Some identities with generalized hypergeometric functions, Appl. Math. Inf. Sci., 10 (2016), 1729-1734. 1

[16] X.-J. Yang, D. Baleanu, H. M. Srivastava, Local fractional integral transforms and their applications, Elsevier/Academic Press, Amsterdam, (2016).

[17] X.-J. Yang, H. M. Srivastava, An asymptotic perturbation solution for a linear oscillator of free damped vibrations in fractal medium described by local fractional derivatives, Commun. Nonlinear Sci. Numer. Simul., 29 (2015), 499-504. 1 